\title{
An Investigation of the Genetic Variability of Metabolic Syndrome and Hemoglobin A1c among African Americans: Rethinking Current Standards of Type 2 Diabetes Diagnosis
}

\author{
Green $\mathrm{RT}^{1}$, Nunlee-Bland $\mathrm{G}^{1}$, Fluitt $\mathrm{MB}^{1}$, Gambhir $\mathrm{KK}^{1^{*}}$ \\ ${ }^{1}$ Division Endocrinology and Metabolism, Department of Medicine, Howard University College of Medicine, Howard University, \\ USA
}

Corresponding Author: Kanwal K. Gambhir, Ph.D ${ }^{\text {ORCID iD }}$

Address: The Division of Endocrinology, Department of Medicine, Howard University College of Medicine, Washington, DC 20060, USA.

Received date: 16 November 2020; Accepted date: 02 January 2021; Published date: 09 January 2021

Citation: Green RT, Nunlee-Bland G, Fluitt MB, Gambhir KK. An Investigation of the Genetic Variability of Metabolic Syndrome and Hemoglobin A1c among African Americans: Rethinking Current Standards of Type 2 Diabetes Diagnosis. Diab Res Open Access. 2021 Jan 09;3(1):1-6.

Copyright (C) 2021 Green RT, Nunlee-Bland G, Fluitt MB, Gambhir KK. This is an open-access article distributed under the Creative Commons Attribution License, which permits unrestricted use, distribution, and reproduction in any medium, provided the original work is properly cited.

\begin{abstract}
Biological markers for Metabolic Syndrome, such as serum lipids and Hemoglobin A1c may have genetic variability among African Americans versus their Caucasian American counterparts. Although cases of Type 2 diabetes and its sequela significantly outweigh Caucasian Americans in the US, paradoxically research has found a lower prevalence of Metabolic Syndrome among blacks versus whites despite there being higher rates of Type 2 diabetes among the former population. Research has shown Metabolic Syndrome lipid parameters among African Americans are more favorable despite outstanding Type 2 diabetes incidence. With the emergence of the Human Genome Project and Genome Wide Association Studies, genetic differences in these parameters have been uncovered and genetic variability may play a role in such paradoxical mismatch. It may be reasonable to consider modifying Metabolic Syndrome parameters as given this new biological evidence. Hemoglobin A1c, also a biological marker used to monitor glucose levels over time, shows variability in its measurements with respect to African Americans. Genetic factors may play a role in the discrepancies among African Americans populations when using this parameter to monitor blood glucose. Precision medicine is now at the forefront of a biomedical new age, to find therapies that cater to specific populations based on genetic research. African Americans may not benefit from such a revolutionary paradigm shift in medicine due to evidence of lack of inclusivity in such studies like the Human Genome Project. Consideration should be made to the future of molecular medicine to include more minority populations such as African Americans in order to cater to specific differences rather than generalized standards of care.
\end{abstract}

\section{Keywords}

Metabolic Syndrome (MetS), Hemoglobin A1c (HbA1c), African Americans (AAs), Type 2 Diabetes (T2DM), Genome Wide Association Studies (GWAS) 


\section{Introduction}

The use of biological markers such as Metabolic Syndrome (MetS) and HbAic have long been accepted as the standard method for diagnosing and treating populations with Type 2 diabetes mellitus (T2DM). However, the paradox of African Americans (AAs) being disproportionately affected by the prevalence of diabetes despite practitioners using these ubiquitous standards to diagnose, manage, and treat patients warrants investigation. It has long been accepted that T2DM is a multifactorial disease with environmental risk factors such as obesity, nutrition and socioeconomic status being labeled as primary reasons for the disproportion of T2DM among AAs. In addition, genetic variability also contributes to the possible inefficacy of these very biological markers used in T2DM diagnosis and management. With the use of Genome Wide Association Studies (GWAS), insight into genetic differences in allelic frequencies and polymorphisms has shown differences in parameters of MetS, namely lipid profiles, and in hemoglobin A1c (HbA1c) levels. Peer reviewed literature is used to investigate these genetic variations in these biomarkers of T2DM and possible implications in their use as a diagnostic tools in diabetic and pre-diabetic AAs.

Metabolic Syndrome paradox among African Americans:

The National Cholesterol Education Program Adult Treatment Panel III (NCEP ATP III) defines MetS as having three or more out of the five following criteria: high blood pressure, central obesity, low high-density lipoprotein (HDL) cholesterol, elevated triglycerides, or fasting hyperglycemia $[1,2]$. Type 2 diabetes is a multifactorial disease with several predisposing risk factors leading to its manifestation. Metabolic Syndrome has been shown by several studies to be a significant risk factor of T2DM, cardiovascular disease and all-cause mortality [3]. The Jackson Health Study (JHS) found that there was a high prevalence of cardiovascular disease (CVD) among AAs with increased odds of this disease with MetS [4]. Moreover, the study implied that having control over MetS would result in significant reduction in CVD among AAs [4]. Metabolic Syndrome has the potential to predict $\mathrm{T} 2 \mathrm{DM}$ and therefore poses as a substantial tool in the management of $\mathrm{T} 2 \mathrm{DM}$ in clinical practice [3] by helping clinicians veer patients from a path towards disease progression. Therefore, the potential for reversibility of pre-diabetes can be optimized with a diagnosis of MetS as a risk factor. The effectiveness of the diagnostic criterion of MetS is thus critical, not only in the diagnosis of this metabolic disorder, but also in the cascade of disorders that may follow, such T2DM.

African Americans have among the highest rates of diabetes alone and diabetes accompanied by its complications such as end-stage renal disease (ESRD), amputation, visual impairment and death [5]. In fact, African Americans are twice as likely as non-Hispanic Caucasians to die from diabetes [5]. Since MetS is a major risk factor of $\mathrm{T} 2 \mathrm{DM}$ and being intrinsically comprised of five criteria, it would be natural to assume that with such high prevalence of T2DM among AAs that all or most of the MetS criteria among AAs would be met. This is surprisingly not the case. Research has found that often there is a lower prevalence of MetS among blacks versus Caucasians despite there being higher rates of T2DM and CVD among blacks [6]. The prevalence of MetS among AA men is actually lower than or equivalent to that seen among those from European ancestry [7]. Therein lays paradoxes among outcomes of African Americans as it pertains to MetS and certain diagnostic criteria of MetS. Based on the heritability of MetS, genetics is a factor worth investigating further.

\section{Serum lipids paradox among African Americans:}

A high triglyceride (TG) and low HDL cholesterol (HDLC) levels nearly meets two of the five prerequisites for a MetS diagnosis. Unexpectedly, AAs generally have healthier lipid profiles (lower TG and higher HDLC concentrations) [7]. According to the NHANES ((National Health and Nutrition Examination Survey) dataset, AAs had one of the lowest mean TG compared to Caucasian and Mexican Americans [7]. Moreover, studies have shown that AAs with insulin resistance have been found to have lower TG and higher HDLC as compared to Caucasians [8]. Serum lipids play a substantial role as a predictive biomarker in cardiometabolic disease and as a result of this more favorable lipid profile among AAs, it would be 
reasonable to assume that T2DM among AAs would be less prevalent, however from an epidemiological standpoint, this is not the observed [7]. The prevalence of T2DM in AAs is more than 1.5 times that of nonHispanic whites [5].

The use of serum lipids in assessing disease risk of MetS is effective for other populations, however, there is a mismatch between the biological markers, TG and HDLC, used to diagnose MetS and T2DM disease among AAs, and according to Rotimi et al, this "metabolic paradox" warrants a discussion of genomic factors, among all variables, that underlay this observation [7].

Heritable traits of Metabolic Syndrome in African Americans:

Heritability of MetS, under the criteria set by the NCEP ATP III is estimated to be approximately 30\%, with individual components higher and having possible variability in ethnicity [9]. Specific blood lipids components have estimated heritability ranging from approximately $35-60 \%$ for HDLC, low density lipoprotein (LDL), and TG blood lipid levels in diabetics [10].

Research has shown variations in genes encoding cholesterol and TG lipids. Meta-analysis of European populations has identified genetic variants in over 150 lipid-associated loci using GWAS [11]. Despite results for additional ethnicities being limited, Lanktree et al identified a novel variation at locus rs868213, in a splice site gene $\left(\mathrm{EXOC}_{3} \mathrm{~L} 1\right)$ that is associated with increased HDLC concentrations using genetic metaanalysis of nearly 16,000 AAs [11]. EXOC3L1 is strongly expressed in vascular endothelium and forms part of a protein complex that is an important facilitator in the trafficking of lipid receptors [11]. Moreover, the minor allele of rs868213 is observed at a much higher rate in studies done with AA cohorts with 43\% versus allele frequencies of $6 \%$ in American populations of European descent [11]. This discovery is one of many novel findings concerning the genetic differences in the makeup of AA lipid profile at a genetic level.

With the emergence of GWAS, numerous genetic loci associated with serum lipid levels have been successfully identified and known to be risk factors for CVD [12]. Although studies have reported significant association of gene loci with these lipid traits in populations of European ancestry, research has shown that there is racial/ethnic variation in blood lipid levels among AAs [13], that is lower TG and higher HDL and these differences seem to have their basis in genetic determinants of lipid profiles of AAs [12]. Based on GWAS studies, genetic variation may be contributing to the different distributions of outcomes of populations from different ethnic backgrounds [12].

\section{Modifying Metabolic Syndrome diagnostic criteria for more inclusivity:}

It is clear that there is a paradoxical lipid profile of AAs with respect to diabetes diagnosis, that is, there is high HDLC and low TG among AAs and AAs with insulin resistance. These two major criterion, TG and HDLC, of MetS may have implications for the diagnosis (or missed diagnosis) of possible individuals at risk of T2DM, among other cardiometabolic diseases. Therefore, at risk AA individuals may not be adequately accounted for by the standard MetS criteria. Moreover, as potential risk factors of T2DM, not acknowledging this discrepancy may continue to lead to overwhelming numbers of increased AA populations with diabetes.

The NCEP ATP III set cutoffs thresholds used to define hypertriglyceridemia (TG level $\geq 150 \mathrm{mg} / \mathrm{dL}$ ) and low HDL levels $(<40 \mathrm{mg} / \mathrm{dL}$ in men and $<50$ $\mathrm{mg} / \mathrm{dL}$ in women) may be not appropriate set points. Since these parameters are used for MetS, the cutoff set for these lipids may in turn lead to underestimation of MetS among AAs [4] and future underestimate potential T2DM cases among AAs. Therefore, the cutoff points currently defined by the MetS guidelines may not be at a level that can identify high risk populations like AAs for T2DM.

Applying uniform cutoff points for TG and HDL cholesterol concentrations for all ethnic populations may results in substantial misclassification [4]. Consideration may need to be made to adjust cut off points by referencing data that is specific to incidence among AAs. Another method would be to use other lipid profiles such as VLDL, LDL concentrations [4] as 
alternative parameters for MetS risk among AAs. This in turn may provide a better estimate of lipid-related risk of MetS among AAs and therefore better suited to help in the prognoses T2DM risk. Therefore, the criteria for MetS may need modification in order to be more inclusive of AA populations.

Discrepancies of $H b A 1 c$ as a marker of $T 2 D M$ among AAs:

African Americans are disproportionately affected by T2DM-associated complications such as lowerextremity amputation, retinopathy, and end-stage renal disease [14]. Glycemic control, therefore, is critical in preventing microvascular complications. Hemoglobin A1c (HbA1c) or glycosylated hemoglobin is a marker used in the diagnosis and monitoring of blood glucose levels in T2DM over a period of time and research has suggested the control of HbA1c shows poorer control among minority populations versus non-minority groups [14].

A genetic variant rs1050828 on the $\mathrm{X}$ chromosome in non-diabetic individuals (all forms) associated with Glucose-6-phosphate dehydrogenase (G6PD) enzyme that is linked with red blood cell parameters that may play a role in reducing red cell life span [15]. G6PD is highly prevalent among AAs with 1 in 10 AAs possessing the genetic deficiency [16].

This G6PD variant poses clinical relevance as this variant would mean HbA1c levels would need to be $0.8 \%$ higher in males versus the current guidelines of $6.5 \%$ in comparison to individuals without the variant, in order to meet the criteria of a T2DM diagnosis [15] This would mean a longer period of underdiagnosed T2DM among AAs, longer exposure of blood glucose and in turn increased risk of long term T2DM complications in AAs versus EAs [15]. Research has shown that a shift from using fasting glucose to HbA1c to diagnose T2DM could result in over $600 \mathrm{~K}$ cases of undiagnosed T2DM AAs with the consideration of this new discovery of this variant allele [15]. Although research has not directly shown these variants in diabetic patients, one could assume that this genetic difference would have a similar effect on those with T2DM [15].
HbA1c is the most common laboratory test performed in people with $\mathrm{T} 2 \mathrm{DM}$ used to measure recent blood glucose levels and is also implemented in T2DM medical interventions and in the assessment of escalation of medical therapy [15]. However, there are often discrepancies found between self-monitored blood glucose and HbA1c in those with T2DM [15].

It has been shown from clinical studies that implemented repeated glucose measures from Continuous Glucose Measurements as compared with concomitant HbA1c from the same individual with T2DM revealed intra-heterogeneity in the relationship between these two measurements and genetic differences may be a prime factor in explaining this variation [15]. Moreover, the genetic influences may play a part in the heterogeneity of intensive therapy outcomes in people with T2DM as some clinical trials have shown [15].

Alternatives for T2DM diagnosis and management have been considered in other glycated proteins such as glycated albumin or fructosamine either by itself or combined with HbA1c [15]. An alternative way to measure blood glucose in AAs should none the less be at least considered based on genetic dissimilarity from non-minority groups based on genetic variations as discussed.

Precision Medicine: Looking into the future of diabetes diagnosis and management:

Advancement in biotechnology using genetics and genomics in the last two decades has made the concept of "Precision Medicine" a new reality. The concept works to find therapies for subgroups of individuals with unique features [17] using advancements specifically in genetic biotechnology which started with the Human Genome Project; the creation of a complete map of the human genome. Using this map, technologies developed to utilize this information birthing the GWAS [17]. The study of genomics has diversified into other technologies such as epigenomics, proteomics, metabolomics, all of which for the purpose of understanding the human genetic makeup for potential use in understanding disease pathways and for future therapies. Hundreds of loci within the human genome have been discovered for 
many complex diseases which have large public health impacts [17].

Personalized medicine in cancer is one example of biomedicine at its best. Tumors have a unique set of genetic and epigenetic profiles and in turn the direct detection of these penetrant somatic mutations in the manifestation of diseased tissue is highly possible [17]. Although the genetic makeup of cancer and T2DM are different, T2DM may be caused by both a combination of genetics and the environment [17], genetics still confers a risk on an individual for T2DM.

Over 100 genetic loci have been identified in GWAS meta-analysis that has significant association with T2DM or related traits [17]. Although genetic risk variants at these loci may only have slight effects on disease predisposition ( $\sim 10-15 \%$ of overall risk), there is still a potential for new therapeutic approaches based on these novel findings [17].

Most novel in this new era is the discovery of disease heterogeneity and possible ethnic specific alleles, made possible by genotyping and sequencing studies in diverse populations [17]. It is clear that diversifying genetic studies with minority populations such as AA may uncover more variation associated with T2DM and its related traits specific to this population. Since T2DM is one of the major causes of morbidity and mortality affecting AAs, it is of even greater importance. Biomarkers used in the diagnosis and management of the disease such as HbA1c and lipid profiles may be a good place to start in the uncovering genetic variations. Biomarkers that are not determined using all risk factors such as genetic determinants of T2DM do not make for all-inclusive parameters, which may in turn exclude minority populations such as AAs rather than help in alleviating the epidemic.

\section{Conclusion}

In conclusion, parameters used in the diagnosis and management of T2DM such lipid and HbA1c biomarkers may not be efficacious when it comes to AAs. African Americans may have genetic risk factors that contribute to their disproportionate numbers of T2DM diagnosis and its accompanied morbidities. Advancements in genetic research using GWAS reveal variation in biological markers specific to AAs that can improve the prognostic and diagnostic value of current clinical markers of T2DM.

It has been well documented that most of the Human Genome Project was undertaken with participants of majority groups such as Caucasian Americans and Asians, with limited genetic profiles collected for minority populations such as AA populations. With all of the advancements occurring in personalized medicine, populations that are most afflicted with disease disparity, as in the case of AAs with T2DM, may have their specific and unique genetic variations unrecognized in turn possible genetic risk factors, diagnostic biomarkers and therapies also overlooked. Even in the $21^{\text {st }}$ century with novel advancements in medical biotechnology there is yet still "genetic disparity" among AAs in the inclusivity of T2DM genetic research. It is important that future genetic and genomic studies include more AA populations to identify additional variants and possible therapeutic advancements unique to this population. Moreover, additional studies in this ethnic group are essential to identify, establish, and improve diagnostic therapeutic efficacy to combat disease progression.

\section{Conflict of Interest}

All authors have read and approved the final version of the manuscript. The authors have no conflicts of interest to declare.

\section{References}

[1] Alberti KG, Zimmet P, Shaw J; IDF Epidemiology Task Force Consensus Group. The metabolic syndrome--a new worldwide definition. Lancet. 2005 Sep 24-30;366(9491):1059-62. [PMID: 16182882]

[2] Grundy SM, Brewer HB Jr, Cleeman JI, Smith SC Jr, Lenfant C; American Heart Association; National Heart, Lung, and Blood Institute. Definition of metabolic syndrome: Report of the National Heart, Lung, and Blood Institute/American Heart Association conference on scientific issues related to definition. Circulation. 2004 Jan 27;109(3):433-38. [PMID: 14744958]

[3] Shin JA, Lee JH, Lim SY, Ha HS, Kwon HS, Park YM, Lee WC, Kang MI, Yim HW, Yoon KH, Son HY. Metabolic syndrome as a predictor of type 2 diabetes, 
Citation: Green RT, Nunlee-Bland G, Fluitt MB, Gambhir KK. An Investigation of the Genetic Variability of Metabolic Syndrome and Hemoglobin A1c among African Americans: Rethinking Current Standards of Type 2 Diabetes Diagnosis. Diab Res Open Access. 2021 Jan 09;3(1):1-6.

Review Article

and its clinical interpretations and usefulness. J Diabetes Investig. $2013 \mathrm{Jul} \mathrm{8;4(4):334-43.} \mathrm{[PMID:}$ 24843675]

[4] Taylor H, Liu J, Wilson G, Golden SH, Crook E, Brunson CD, Steffes M, Johnson WD, Sung JH. Distinct component profiles and high risk among African Americans with metabolic syndrome: the Jackson Heart Study. Diabetes Care. 2008 Jun;31(6):1248-53. PMID: 18332154]

[5] U.S. Department of Health and Human Services Office of Minority Health. Diabetes and African Americans; 2019. Available from:

https://minorityhealth.hhs.gov/omh/browse.aspx?lvl= 4\&lvlid $=18$

[6] Musani SK, Martin LJ, Woo JG, Olivier M, Gurka MJ, DeBoer MD. Heritability of the Severity of the Metabolic Syndrome in Whites and Blacks in 3 Large Cohorts. Circ Cardiovasc Genet. 2017 Apr;10(2):eoo1621. [PMID: 28408709]

[7] Bentley AR, Rotimi CN. Interethnic Differences in Serum Lipids and Implications for Cardiometabolic Disease Risk in African Ancestry Populations. Glob Heart. 2017 Jun;12(2):141-50. [PMID: 28528248]

[8] Gaillard TR. The Metabolic Syndrome and Its Components in African-American Women: Emerging Trends and Implications. Front Endocrinol (Lausanne). 2018 Jan 22;8:383. [PMID: 29403438]

[9] Carty CL, Bhattacharjee S, Haessler J, Cheng I, Hindorff LA, Aroda V, Carlson CS, Hsu CN, Wilkens L, Liu S, Selvin E, Jackson R, North KE, Peters U, Pankow JS, Chatterjee N, Kooperberg C. Analysis of metabolic syndrome components in $>15$ ooo african americans identifies pleiotropic variants: results from the population architecture using genomics and epidemiology study. Circ Cardiovasc Genet. 2014 Aug;7(4):505-13. [PMID: 25023634]

[10] Adebamowo SN, Tekola-Ayele F, Adeyemo AA, Rotimi CN. Genomics of Cardiometabolic Disorders in
Sub-Saharan Africa. Public Health Genomics. 2017;20(1):9-26. [PMID: 28482349]

[11] Lanktree MB, Elbers CC, Li Y, Zhang G, Duan Q, Karczewski KJ, Guo Y, Tragante V, North KE, Cushman M, Asselbergs FW, Wilson JG, Lange LA, Drenos F, Reiner AP, Barnes MR, Keating BJ. Genetic metaanalysis of 15,901 African Americans identifies variation in EXOC3L1 is associated with HDL concentration. J Lipid Res. 2015 Sep;56(9):1781-86. [PMID: 26199122]

[12] Chang MH, Ned RM, Hong Y, Yesupriya A, Yang Q, Liu T, Janssens AC, Dowling NF. Racial/ethnic variation in the association of lipid-related genetic variants with blood lipids in the US adult population. Circ Cardiovasc Genet. 2011 Oct;4(5):523-33. [PMID: 21831959]

[13] Sumner AE. Ethnic differences in triglyceride levels and high-density lipoprotein lead to underdiagnosis of the metabolic syndrome in black children and adults. J Pediatr. 2009 Sep;155(3):S7.e711. [PMID: 19732569]

[14] Kirk JK, D'Agostino RB Jr, Bell RA, Passmore LV, Bonds DE, Karter AJ, Narayan KM. Disparities in HbA1c levels between African-American and non-Hispanic white adults with diabetes: a meta-analysis. Diabetes Care. 2006 Sep;29(9):2130-36. [PMID: 16936167]

[15] Paterson AD. HbA1c for type 2 diabetes diagnosis in Africans and African Americans: Personalized medicine NOW! PLoS Med. 2017 Sep 12;14(9):e1002384. [PMID: 28898251]

[16] MedlinePlus. G6PD Deficiency (Glucose-6phosphate dehydrogenase deficiency). U.S. National Library of Medicine;2020. Available from: https://medlineplus.gov/g6pddeficiency.html

[17] Merino J, Florez JC. Precision medicine in diabetes: an opportunity for clinical translation. Ann N Y Acad Sci. 2018 Jan;1411(1):140-52. [PMID: 29377200]

Keywords: Metabolic Syndrome (MetS), Hemoglobin A1c (HbA1c), African Americans (AAs), Type 2 Diabetes (T2DM), Genome Wide Association Studies (GWAS) 